\title{
ROUGH PRIME IDEALS AND ROUGH FUZZY PRIME IDEALS IN GAMMA-SEMIGROUPS
}

\author{
RONNASON CHINRAM
}

\begin{abstract}
The notion of rough sets was introduced by Z. Pawlak in the year 1982. The notion of a $\Gamma$-semigroup was introduced by M. K. Sen in the year 1981. In 2003, Y. B. Jun studied the roughness of subГsemigroups, ideals and bi-ideals in $\Gamma$-semigroups. In this paper, we study rough prime ideals and rough fuzzy prime ideals in $\Gamma$-semigroups.
\end{abstract}

\section{Introduction}

The notion of rough sets was introduced by Z. Pawlak in the year 1982 ([14]). The theory of rough sets has emerged as another major mathematical approach for managing uncertainty that arises from inexact, noisy or incomplete information. The algebraic approach of rough sets was studied by some authors, for example, J. Iwinski ([7]) and N. Kuroki ([10], [11], and [12]) studied algebraic properties of rough sets; R. Biswas and S. Nanda ([2]) introduced the notion of rough subgroups; B. Davvaz ([6]) studied rough subpolygroups in a factor polygroup; and Q. M. Xiao and Z. L. Zhang ([20]) studied rough prime ideals and rough fuzzy prime ideals in semigroups. The notion of a $\Gamma$-semigroup was introduced by M. K. Sen in the year 1981 ([17]). Г-semigroups generalize semigroups. Many classical notions of semigroups have been extended to $\Gamma$-semigroups (see [3], [4], [5], [15], [16], [17], and [18]). Some properties of $\Gamma$-semigroups were studied by some authors, for example, the author ([3] and [4]) studied quasi-ideals and bi-ideals in $\Gamma$-semigroups; Y. I. Kwon and S. K. Lee ([13]) studied weakly prime ideals of ordered $\Gamma$-semigroups; Y. B. Jun ([9]) studied closure $\Gamma$-semigroups and M. Siripitukdet and A. Iampan ([19]) studied the ordered $n$-prime ideals in ordered $\Gamma$-semigroups. In 2003, Y. B. Jun ([8]) studied the roughness of sub $\Gamma$-semigroups, ideals and bi-ideals in $\Gamma$-semigroups.

Received August 22, 2008; Revised January 23, 2009.

2000 Mathematics Subject Classification. Primary 20N25, 20M99, 20M12.

Key words and phrases. congruences, $\Gamma$-semigroups, quotient $\Gamma$-semigroups, prime ideals, $\Theta$-lower rough prime ideals, $\Theta$-upper rough prime ideals.

This work was financially supported by the Commission on Higher Education and the Thailand Research Fund (TRF) - MRG5080220. 
In this paper, we study $\Theta$-lower and $\Theta$-upper rough prime ideals and $\Theta$-lower and $\Theta$-upper rough fuzzy prime ideals in $\Gamma$-semigroups.

\section{Preliminaries}

Let $S=\{x, y, z, \ldots\}$ and $\Gamma=\{\alpha, \beta, \gamma, \ldots\}$ be two nonempty sets. $S$ is called a $\Gamma$-semigroup if $S$ satisfies the identities $x \gamma y \in S$ and $(x \gamma y) \mu z=x \gamma(y \mu z)$ for all $x, y, z \in S$ and $\gamma, \mu \in \Gamma$.

Let $S$ be an arbitrary semigroup and $\Gamma$ any nonempty set. Let $a \gamma b=a b$ for all $a, b \in S$ and $\gamma \in \Gamma$. It is easy to see that $S$ is a $\Gamma$-semigroup. Thus a semigroup can be considered to be a $\Gamma$-semigroup.

Let $S$ be a $\Gamma$-semigroup and $\alpha$ a fixed element in $\Gamma$. We define $a \cdot b=a \alpha b$ for all $a, b \in S$. We can show that $(S, \cdot)$ is a semigroup.

Let $S$ be a $\Gamma$-semigroup. A nonempty subset $T$ of $S$ is called a $s u b \Gamma$ semigroup of $S$ if $a \gamma b \in T$ for all $a, b \in T$ and $\gamma \in \Gamma$. A nonempty subset $L$ of $S$ is called a left ideal of $S$ if $S \Gamma L \subseteq L$ and a nonempty subset $R$ of $S$ is called a right ideal of $S$ if $R \Gamma S \subseteq R$. A nonempty subset $I$ of $S$ is called an ideal of $S$ if $I$ is both a left and a right ideal of $S$.

Let $S$ be a $\Gamma$-semigroup. An equivalence relation $\Theta$ is called a congruence on $S$ if for all $a, b, x \in S$ and $\gamma \in \Gamma$, if $(a, b) \in \Theta$, then $(a \gamma x, b \gamma x) \in \Theta$ and $(x \gamma a, x \gamma b) \in \Theta$. Let $[a]_{\Theta}$ denote the congruence class containing the element $a \in S$. A congruence $\Theta$ on $S$ is said to be complete if $[a]_{\Theta} \gamma[b]_{\Theta}=[a \gamma b]_{\Theta}$ for all $a, b \in S$ and $\gamma \in \Gamma$. Let $A$ be a nonempty subset of $S$ and $\Theta$ a congruence on $S$. The $\Theta$-lower approximation and $\Theta$-upper approximation of $A$ are defined to be the sets

$$
\underline{\Theta}(A)=\left\{x \in S \mid[x]_{\Theta} \subseteq A\right\}
$$

and

$$
\bar{\Theta}(A)=\left\{x \in S \mid[x]_{\Theta} \cap A \neq \emptyset\right\},
$$

respectively. Let $\Theta$ be a congruence on a $\Gamma$-semigroup $S$. A nonempty subset $A$ of $S$ is called a $\Theta$-lower $(\Theta$-upper, respectively) rough subГ-semigroup of $S$ if the $\Theta$-lower ( $\Theta$-upper, respectively) approximation of $A$ is a sub $\Gamma$-semigroup of $S$. The $\Theta$-lower and $\Theta$-upper rough right ideal (left ideal, ideal) of $S$ are defined analogously. The following theorem is well-known ([8]).

Theorem 2.1. Let $\Theta$ be a complete congruence on a $\Gamma$-semigroup $S$ and $I$ an ideal of $S$. Then the following statements hold.

(i) If $\underline{\Theta}(I) \neq \emptyset$, then $I$ is a $\Theta$-lower rough ideal of $S$.

(ii) $I$ is a $\Theta$-upper rough ideal of $S$.

\section{Rough prime ideals}

An ideal $I$ of a $\Gamma$-semigroup $S$ is a prime ideal of $S$ if for $x, y \in S$ and $\gamma \in \Gamma$ if $x \gamma y \in I$ implies $x \in I$ or $y \in I$. Let $\Theta$ be a congruence on a $\Gamma$-semigroup $S$. Then a subset $A$ of $S$ is called a $\Theta$-lower rough prime ideal of $S$ if $\underline{\Theta}(A)$ is a prime ideal of $S$. A $\Theta$-upper rough prime ideal of $S$ is defined analogously. $A$ 
is called a rough prime ideal of $R$ if $A$ is a $\Theta$-lower and $\Theta$-upper rough prime ideal of $S$.

Example 3.1. Let $\Gamma=\{5,7\}$. For $x, y \in \mathbb{N}$ and $\gamma \in \Gamma$, define $x \gamma y=x \cdot \gamma \cdot y$ where $\cdot$ is the usual multiplication on $\mathbb{N}$. Then $\mathbb{N}$ is a $\Gamma$-semigroup.

Define a relation $\Theta$ on $\mathbb{N}$ by

$$
x \Theta y \Leftrightarrow 3 \mid x-y \text { for all } x, y \in \mathbb{N} .
$$

Then $\Theta$ is a congruence on $\mathbb{N}$ such that $\Theta$-congruence classes are the subset $\{1,4,7,10, \ldots\}=\{3 n-2 \mid n \in \mathbb{N}\},\{2,5,8,11, \ldots\}=\{3 n-1 \mid n \in \mathbb{N}\}$ and $\{3,6,9,12, \ldots\}=\{3 n \mid n \in \mathbb{N}\}$.

Let $A_{1}=\{3,12\}$. We have $\underline{\Theta}\left(A_{1}\right)=\emptyset$ and $\bar{\Theta}\left(A_{1}\right)=\{3 n \mid n \in \mathbb{N}\}$. So $\underline{\Theta}\left(A_{1}\right)$ is not a prime ideal of $\mathbb{N}$. Next, we claim that $\bar{\Theta}\left(A_{1}\right)$ is a prime ideal of $\mathbb{N}$. It is easy to see that $\bar{\Theta}\left(A_{1}\right)=\{3 n \mid n \in \mathbb{N}\}$ is an ideal of a $\Gamma$-semigroup $\mathbb{N}$. Let $x, y \in \mathbb{N}$ such that $x \gamma y \in \bar{\Theta}\left(A_{1}\right)$. Then $3 \mid x \gamma y$. Since $3 \nmid \gamma$, we have $3 \mid x$ or $3 \mid y$. Thus $x \in \bar{\Theta}\left(A_{1}\right)$ or $y \in \bar{\Theta}\left(A_{1}\right)$. So $A_{1}$ is a $\Theta$-upper rough prime ideal of $\mathbb{N}$ but not a $\Theta$-lower rough prime ideal of $\mathbb{N}$.

Let $A_{2}=\{3 n \mid n \in \mathbb{N}\} \cup\{1\}$. We have $\underline{\Theta}\left(A_{2}\right)=\{3 n \mid n \in \mathbb{N}\}$ and $\bar{\Theta}\left(A_{2}\right)=\{3 n \mid n \in \mathbb{N}\} \cup\{3 n-2 \mid n \in \mathbb{N}\}$. Then $\underline{\Theta}\left(A_{2}\right)$ is a prime ideal of $\mathbb{N}$. But $\bar{\Theta}\left(A_{2}\right)$ is not a ideal of $\mathbb{N}$ because $10=2 \cdot 5 \cdot 1 \in \mathbb{N} \Gamma \bar{\Theta}\left(A_{2}\right)$ but $10 \notin \bar{\Theta}\left(A_{2}\right)$. So $A_{2}$ is a $\Theta$-lower rough prime ideal of $\mathbb{N}$ but not a $\Theta$-upper rough prime ideal of $\mathbb{N}$.

Let $A_{3}=\{3 n \mid n \in \mathbb{N}\} \cup\{1,2\}$. We have $\underline{\Theta}\left(A_{3}\right)=\{3 n \mid n \in \mathbb{N}\}$ and $\bar{\Theta}\left(A_{3}\right)=\mathbb{N}$. Then $\underline{\Theta}\left(A_{3}\right)$ and $\bar{\Theta}\left(A_{3}\right)=\mathbb{N}$ are prime ideals of $\mathbb{N}$. Therefore $A_{3}$ is a $\Theta$-lower rough prime ideal of $\mathbb{N}$ and a $\Theta$-upper rough prime ideal of $\mathbb{N}$. Then $A_{3}$ is a rough prime ideal of $\mathbb{N}$.

If $A_{4}=\{2 n \mid n \in \mathbb{N}\}$, then $A_{4}$ is a prime ideal of $\mathbb{N}$ but $\underline{\Theta}\left(A_{4}\right)=\emptyset$. Therefore if $I$ is a prime ideal, then $\underline{\Theta}(I)$ need not be a prime ideal.

The following theorem is true.

Theorem 3.1. Let $\Theta$ be a complete congruence on a $\Gamma$-semigroup $S$ and $I$ a prime ideal of $S$. The following statements are true.

(i) If $\underline{\Theta}(I) \neq \emptyset$, then $I$ is a $\Theta$-lower rough prime ideal of $S$.

(ii) $I$ is a $\Theta$-upper rough prime ideal of $S$.

(iii) If $\underline{\Theta}(I) \neq \emptyset$, then $I$ is a rough prime ideal of $S$.

Proof. (i) By Theorem 2.1(i), we know that $\underline{\Theta}(I)$ is an ideal of $S$. We suppose that $\underline{\Theta}(I)$ is not prime. Then there exist $x, y \in S$ and $\gamma \in \Gamma$ such that $x \gamma y \in$ $\Theta(I)$ but $x \notin \Theta(I)$ and $y \notin \Theta(I)$. Thus $[x]_{\Theta} \not \subset I$ and $[y]_{\Theta} \not \subset I$. Then there exists $a \in[x]_{\Theta}$ but $a \notin I$ and there exists $b \in[y]_{\Theta}$ but $b \notin I$. We have $a \gamma b \in[x]_{\Theta} \gamma[y]_{\Theta} \subseteq[x \gamma y]_{\Theta}$. Since $x \gamma y \in \underline{\Theta}(I),[x \gamma y]_{\Theta} \subseteq I$. This implies $a \gamma b \in I$. Since $I$ is a prime ideal of $S, a \in I$ or $b \in I$. It contradicts the supposition. Therefore $\underline{\Theta}(I)$ is a prime ideal of $S$. Hence $I$ is a $\Theta$-lower rough prime ideal of $S$. 
(ii) By Theorem 2.1(ii), we know that $\bar{\Theta}(I)$ is an ideal of $S$. Let $x, y \in S$ and $\gamma \in \Gamma$ such that $x \gamma y \in \bar{\Theta}(I)$. Then $\left([x]_{\Theta} \gamma[y]_{\Theta}\right) \cap I=[x \gamma y]_{\Theta} \cap I \neq \emptyset$. Thus there exist $a \in[x]_{\Theta}$ and $b \in[y]_{\Theta}$ such that $a \gamma b \in I$. Since $I$ is prime, $a \in I$ or $b \in I$. Thus $[x]_{\Theta} \cap I \neq \emptyset$ or $[y]_{\Theta} \cap I \neq \emptyset$. So $x \in \bar{\Theta}(I)$ or $y \in \bar{\Theta}(I)$. Therefore $\bar{\Theta}(I)$ is a prime ideal of $S$. Hence $I$ is a $\Theta$-upper rough prime ideal of $S$.

(iii) It follows from (i) and (ii).

However, the converse of Theorem 3.1 is not true in general. From Example 3.1, we can see that $A_{3}$ is not a prime ideal of $\mathbb{N}$ but $A_{3}$ is a $\Theta$-lower rough prime ideal of $\mathbb{N}$ and a $\Theta$-upper rough prime ideal of $\mathbb{N}$.

Let $S$ be a $\Gamma$-semigroup and $\Theta$ a congruence on $S$. Let

$$
S / \Theta=\left\{[x]_{\Theta} \mid x \in S\right\} .
$$

For $[a]_{\Theta},[b]_{\Theta} \in S / \Theta$ and $\gamma \in \Gamma$, let $[a]_{\Theta} \gamma[b]_{\Theta}=[a \gamma b]_{\Theta}$. This is well-defined, since for all $a, a^{\prime}, b, b^{\prime} \in S$ and $\gamma \in \Gamma$,

$$
\begin{aligned}
{[a]_{\Theta}=\left[a^{\prime}\right]_{\Theta} \text { and }[b]_{\Theta}=\left[b^{\prime}\right]_{\Theta} } & \Rightarrow\left(a, a^{\prime}\right),\left(b, b^{\prime}\right) \in \Theta \\
& \Rightarrow\left(a \gamma b, a^{\prime} \gamma b\right),\left(a^{\prime} \gamma b, a^{\prime} \gamma b^{\prime}\right) \in \Theta \\
& \Rightarrow\left(a \gamma b, a^{\prime} \gamma b^{\prime}\right) \in \Theta \\
& \Rightarrow[a \gamma b]_{\Theta}=\left[a^{\prime} \gamma b^{\prime}\right]_{\Theta} .
\end{aligned}
$$

Let $a, b, c \in S$ and $\gamma, \mu \in \Gamma$. We have

$$
\left([a]_{\Theta} \gamma[b]_{\Theta}\right) \mu[c]_{\Theta}=[((a \gamma b) \mu c)]_{\Theta}=[(a \gamma(b \mu c))]_{\Theta}=[a]_{\Theta} \gamma\left([b]_{\Theta} \mu[c]_{\Theta}\right) .
$$

Then the quotient set $S / \Theta$ is a $\Gamma$-semigroup. It is called a quotient $\Gamma$-semigroup of $S$ by $\Theta$. We can see some properties of quotient $\Gamma$-semigroups in [5].

Let $\Theta$ be a congruence on a $\Gamma$-semigroup $S$. The $\Theta$-lower approximation and $\Theta$-upper approximation can be presented in an equivalent form as shown below:

and

$$
\underline{\Theta}(A) / \Theta=\left\{[x]_{\Theta} \in S / \Theta \mid[x]_{\Theta} \subseteq A\right\}
$$

respectively.

$$
\bar{\Theta}(A) / \Theta=\left\{[x]_{\Theta} \in S / \Theta \mid[x]_{\Theta} \cap A \neq \emptyset\right\},
$$

Lemma 3.2. Let $\Theta$ be a complete congruence on a $\Gamma$-semigroup $S$. The following statements are true.

(i) If $I$ is a $\Theta$-lower rough ideal of $S$, then $\underline{\Theta}(I) / \Theta$ is an ideal of $S / \Theta$.

(ii) If $I$ is a $\Theta$-upper rough ideal of $S$, then $\bar{\Theta}(I) / \Theta$ is an ideal of $S / \Theta$.

Proof. (i) Let $[x]_{\Theta} \in \underline{\Theta}(I) / \Theta,[y]_{\Theta} \in S / \Theta$ and $\gamma \in \Gamma$. Then $[x]_{\Theta} \subseteq I$. This implies $x \in \underline{\Theta}(I)$. Since $\underline{\Theta}(I)$ is an ideal of $S, x \gamma y \in \underline{\Theta}(I)$. Then $[x \gamma y]_{\Theta} \subseteq I$. We have

$$
[x]_{\Theta} \gamma[y]_{\Theta}=[x \gamma y]_{\Theta} \subseteq I .
$$

Then $[x]_{\Theta} \gamma[y]_{\Theta} \in \underline{\Theta}(I) / \Theta$. Similarly, $[y]_{\Theta} \gamma[x]_{\Theta} \in \underline{\Theta}(I) / \Theta$. Therefore $\underline{\Theta}(I) / \Theta$ is an ideal of $S / \Theta$. 
(ii) Let $[x]_{\Theta} \in \bar{\Theta}(I) / \Theta,[y]_{\Theta} \in S / \Theta$ and $\gamma \in \Gamma$. Then $[x]_{\Theta} \cap I \neq \emptyset$. This implies $x \in \bar{\Theta}(I)$. Since $\bar{\Theta}(I)$ is an ideal of $S, x \gamma y \in \bar{\Theta}(I)$. Then $[x \gamma y]_{\Theta} \cap I \neq \emptyset$. We have

$$
\left([x]_{\Theta} \gamma[y]_{\Theta}\right) \cap I=[x \gamma y]_{\Theta} \cap I \neq \emptyset .
$$

Then $[x]_{\Theta} \gamma[y]_{\Theta} \in \bar{\Theta}(I) / \Theta$. Similarly, $[y]_{\Theta} \gamma[x]_{\Theta} \in \bar{\Theta}(I) / \Theta$. Therefore $\bar{\Theta}(I) / \Theta$ is an ideal of $S / \Theta$.

Theorem 3.3. Let $\Theta$ be a complete congruence on a $\Gamma$-semigroup $S$. The following statements are true.

(i) If $I$ is a $\Theta$-lower rough prime ideal of $S$, then $\underline{\Theta}(I) / \Theta$ is a prime ideal of $S / \Theta$.

(ii) If I is a $\Theta$-upper rough prime ideal of $S$, then $\bar{\Theta}(I) / \Theta$ is a prime ideal of $S / \Theta$.

Proof. (i) By Lemma 3.2(i), we know that $\underline{\Theta}(I) / \Theta$ is an ideal of $S$. Let $[x]_{\Theta},[y]_{\Theta} \in S / \Theta$ and $\gamma \in \Gamma$ such that $[x]_{\Theta} \gamma[y]_{\Theta} \in \underline{\Theta}(I) / \Theta$. Then $[x \gamma y]_{\Theta} \in$ $\underline{\Theta}(I) / \Theta$. This implies $[x \gamma y]_{\Theta} \subseteq I$. Then $x \gamma y \in \underline{\Theta}(I)$. Since $I$ is a $\Theta$-lower rough prime ideal of $S, x \in \underline{\Theta}(I)$ or $y \in \underline{\Theta}(I)$. So $[x]_{\Theta} \subseteq I$ or $[y]_{\Theta} \subseteq I$. Thus $[x]_{\Theta} \in \underline{\Theta}(I) / \Theta$ or $[y]_{\Theta} \in \underline{\Theta}(I) / \Theta$. Therefore $\underline{\Theta}(I) / \Theta$ is a prime ideal of $S / \Theta$.

(ii) By Lemma 3.2(ii), we know that $\bar{\Theta}(I) / \Theta$ is an ideal of $S$. Let $[x]_{\Theta},[y]_{\Theta} \in$ $S / \Theta$ and $\gamma \in \Gamma$ such that $[x]_{\Theta} \gamma[y]_{\Theta} \in \bar{\Theta}(I) / \Theta$. Then $[x \gamma y]_{\Theta} \in \bar{\Theta}(I) / \Theta$. Thus $[x \gamma y]_{\Theta} \cap I \neq \emptyset$. Then $x \gamma y \in \bar{\Theta}(I)$. Since $I$ is a $\Theta$-upper rough prime ideal of $S, x \in \bar{\Theta}(I)$ or $y \in \bar{\Theta}(I)$. So $[x]_{\Theta} \cap I \neq \emptyset$ or $[y]_{\Theta} \cap I \neq \emptyset$. Then $[x]_{\Theta} \in \bar{\Theta}(I) / \Theta$ or $[y]_{\Theta} \in \bar{\Theta}(I) / \Theta$. Hence $\bar{\Theta}(I) / \Theta$ is a prime ideal of $S / \Theta$.

\section{Rough fuzzy prime ideals}

A function $f$ from $S$ to the unit interval $[0,1]$ is called a fuzzy subset of $S$. The $\Gamma$-semigroup $S$ itself is a fuzzy subset of $S$ such that $S(x)=1$ for all $x \in S$, denoted also by $S$. A fuzzy subset $f$ of a $\Gamma$-semigroup $S$ is called a fuzzy ideal of $S$ if $f(x \gamma y) \geq f(x) \vee f(y)$ for any $x, y \in S$ and $\gamma \in \Gamma$.

Let $f$ be a fuzzy subset of $S$ and $\lambda \in[0,1]$. The set

$$
f_{\lambda}=\{x \in S \mid f(x) \geq \lambda\}
$$

is called the $\lambda$-level set of a fuzzy set $f$.

Theorem 4.1. Let $f$ be a fuzzy subset of a $\Gamma$-semigroup $S$. Then $f$ is a fuzzy ideal of $S$ if and only if for all $\lambda \in[0,1]$, if $f_{\lambda} \neq \emptyset$, then $f_{\lambda}$ is an ideal of $S$.

Proof. Assume $f$ is a fuzzy ideal of $S$. Then $f(x \gamma y) \geq f(x) \vee f(y)$ for any $x, y \in S$ and $\gamma \in \Gamma$. Assume $f_{\lambda} \neq \emptyset$. Let $x \in f_{\lambda}, y \in S$ and $\gamma \in \Gamma$. Thus $f(x) \geq \lambda$. Since $f$ is a fuzzy ideal of $S, f(x \gamma y) \geq f(x) \vee f(y) \geq f(x) \geq \lambda$. Therefore $x \gamma y \in f_{\lambda}$. Similarly, $y \gamma x \in f_{\lambda}$. Hence $f_{\lambda}$ is an ideal of $S$.

Conversely, assume for all $\lambda \in[0,1]$, if $f_{\lambda} \neq \emptyset$, then $f_{\lambda}$ is an ideal of $S$. Let $x, y \in S$ and $\gamma \in \Gamma$. 
Case 1: $f(x) \geq f(y)$. Let $\lambda=f(x)$. Then $x \in f_{\lambda}$. By assumption, we have $f_{\lambda}$ is an ideal of $S$. So $x \gamma y \in f_{\lambda}$. Then $f(x \gamma y) \geq \lambda=f(x)=f(x) \vee f(y)$.

Case 2 : $f(x)<f(y)$. Let $\lambda=f(y)$. Then $y \in f_{\lambda}$. By assumption, we have $f_{\lambda}$ is an ideal of $S$. So $x \gamma y \in f_{\lambda}$. Then $f(x \gamma y) \geq \lambda=f(y)=f(x) \vee f(y)$.

Therefore $f$ is a fuzzy ideal of $S$.

Let $f$ be a fuzzy subset of $S$ and $\lambda \in[0,1]$. The set

$$
f_{\lambda}^{S}=\{x \in S \mid f(x)>\lambda\}
$$

is called the $\lambda$-strong level set of a fuzzy set $f$.

Theorem 4.2. Let $f$ be a fuzzy subset of a $\Gamma$-semigroup $S$. Then $f$ is a fuzzy ideal of $S$ if and only if for all $\lambda \in[0,1]$, if $f_{\lambda}^{S} \neq \emptyset$, then $f_{\lambda}^{S}$ is an ideal of $S$.

Proof. Assume $f$ is a fuzzy ideal of $S$. Then $f(x \gamma y) \geq f(x) \vee f(y)$ for any $x, y \in S$ and $\gamma \in \Gamma$. Assume $f_{\lambda}^{S} \neq \emptyset$. Let $x \in f_{\lambda}^{S}, y \in S$ and $\gamma \in \Gamma$. Thus $f(x)>\lambda$. Since $f$ is a fuzzy ideal of $S, f(x \gamma y) \geq f(x) \vee f(y) \geq f(x)>\lambda$. Therefore $x \gamma y \in f_{\lambda}^{S}$. Similarly, $y \gamma x \in f_{\lambda}^{S}$. Hence $f_{\lambda}^{S}$ is an ideal of $S$.

Conversely, assume for all $\lambda \in[0,1]$, if $f_{\lambda}^{S} \neq \emptyset$, then $f_{\lambda}^{S}$ is an ideal of $S$. Let $x, y \in S$ and $\gamma \in \Gamma$.

Case $1: f(x) \geq f(y)$. Thus $x \in f_{\lambda}^{S}$ for all $\lambda<f(x)$. By assumption, we have $f_{\lambda}^{S}$ is an ideal of $S$ for all $\lambda<f(x)$. So $x \gamma y \in f_{\lambda}^{S}$ for all $\lambda<f(x)$. Then $f(x \gamma y)>\lambda$ for all $\lambda<f(x)$. Then $f(x \gamma y) \geq f(x)=f(x) \vee f(y)$.

Case 2 : $f(x)<f(y)$. Then $y \in f_{\lambda}^{S}$ for all $\lambda<f(y)$. By assumption, we have $f_{\lambda}^{S}$ is an ideal of $S$ for all $\lambda<f(y)$. So $x \gamma y \in f_{\lambda}^{S}$ for all $\lambda<f(y)$. Then $f(x \gamma y)>\lambda$ for all $\lambda<f(y)$. Then $f(x \gamma y) \geq f(y)=f(x) \vee f(y)$.

Therefore $f$ is a fuzzy ideal of $S$.

Let $f$ be a fuzzy subset of $S$. Let $\underline{\Theta}(f)$ and $\bar{\Theta}(f)$ be fuzzy subsets of $S$ defined by

$$
\underline{\Theta}(f)(x)=\bigwedge_{a \in[x]_{\Theta}} f(a) \text { and } \bar{\Theta}(f)(x)=\bigvee_{a \in[x]_{\Theta}} f(a) .
$$

The fuzzy subsets $\underline{\Theta}(f)$ and $\bar{\Theta}(f)$ of $S$ are called, respectively, the $\Theta$-lower approximation and $\Theta$-upper approximation of a fuzzy set $f$.

Example 4.1. Let $\mathbb{N}$ be a $\Gamma$-semigroup by $\Gamma=\{5,7\}$ and $x \gamma y=x \cdot \gamma \cdot y$ where - is the usual multiplication on $\mathbb{N}$. Define a congruence $\Theta$ on $\mathbb{N}$ by

$$
x \Theta y \Leftrightarrow 3 \mid x-y \text { for all } x, y \in \mathbb{N} .
$$

Let $f_{1}: \mathbb{N} \rightarrow[0,1]$ by

$$
f_{1}(n)=\frac{1}{n} \text { for all } n \in \mathbb{N} \text {. }
$$


We have that $\underline{\Theta}\left(f_{1}\right)(n)=0$ for all $n \in \mathbb{N}$. Then $\underline{\Theta}\left(f_{1}\right)$ is a fuzzy ideal of $\mathbb{N}$. We have that

$$
\bar{\Theta}\left(f_{1}\right)(n)= \begin{cases}1 & \text { if } n \equiv 1(\bmod 3), \\ \frac{1}{2} & \text { if } n \equiv 2(\bmod 3), \\ \frac{1}{3} & \text { if } n \equiv 0(\bmod 3) .\end{cases}
$$

It is easy to see that $f_{1}$ and $\bar{\Theta}\left(f_{1}\right)$ are not fuzzy ideals of $\mathbb{N}$.

Let $f_{2}: \mathbb{N} \rightarrow[0,1]$ by

$$
f_{2}(n)=1-\frac{1}{n} \text { for all } n \in \mathbb{N} .
$$

It is easy to see that $f_{2}$ is a fuzzy ideal of $\mathbb{N}$. We have that $\bar{\Theta}\left(f_{2}\right)(n)=1$ for all $n \in \mathbb{N}$. Then $\bar{\Theta}\left(f_{2}\right)$ is a fuzzy ideal of $\mathbb{N}$. We have that

$$
\underline{\Theta}\left(f_{2}\right)(n)= \begin{cases}0 & \text { if } n \equiv 1(\bmod 3), \\ \frac{1}{2} & \text { if } n \equiv 2(\bmod 3), \\ \frac{2}{3} & \text { if } n \equiv 0(\bmod 3) .\end{cases}
$$

It is easy to see that $\underline{\Theta}\left(f_{2}\right)$ is not a fuzzy ideal of $\mathbb{N}$.

Let $\Theta$ be a complete congruence on a $\Gamma$-semigroup $S$ and $A$ a nonempty subset of $S$. The characteristic mapping of $A$, denoted by $\chi_{A}$, is the mapping of $S$ into [0,1] defined by

$$
\chi_{A}(x)= \begin{cases}1 & \text { if } x \in A, \\ 0 & \text { if } x \notin A .\end{cases}
$$

Theorem 4.3. Let $\Theta$ be a complete congruence on a $\Gamma$-semigroup $S$ and $A$ a nonempty subset of $S$. The following statements are true.

(i) $A$ is a $\Theta$-lower rough ideal of $S$ if and only if $\underline{\Theta}\left(\chi_{A}\right) \neq 0$ and $\underline{\Theta}\left(\chi_{A}\right)$ is a fuzzy ideal of $S$.

(ii) $A$ is a $\Theta$-upper rough ideal of $S$ if and only if $\bar{\Theta}\left(\chi_{A}\right)$ is a fuzzy ideal of $S$.

Proof. (i) Assume $A$ is a $\Theta$-lower rough ideal of $S$. So $\underline{\Theta}(A)$ is an ideal of $S$. Then there exists $a \in S$ such that $[a]_{\Theta} \subseteq A$. This implies $\underline{\Theta}\left(\chi_{A}\right) \neq 0$. Let $x, y \in S$ and $\gamma \in \Gamma$. We claim that $\bigwedge_{\substack{c \in[x]_{\Theta} \\ d \in[y]_{\Theta}}} \chi_{A}(c \gamma d) \geq\left(\bigwedge_{c \in[x]_{\Theta}} \chi_{A}(c)\right) \vee$ $\left(\bigwedge_{d \in[y]_{\Theta}} \chi_{A}(d)\right)$

Case 1: $[x]_{\Theta} \subseteq A$ or $[y]_{\Theta} \subseteq A$. Then $c \in \underline{\Theta}(A)$ for all $c \in[x]_{\Theta}$ or $d \in \underline{\Theta}(A)$ for all $d \in[y]_{\Theta}$. Since $\underline{\Theta}(A)$ is an ideal of $S, c \gamma d \in \underline{\Theta}(A)$ for all $c \in[x]_{\Theta}$ and $d \in[y]_{\Theta}$. Then $\bigwedge_{\substack{c \in[x]_{\Theta} \\ d \in[y]_{\Theta}}} \chi_{A}(c \gamma d)=1$. This implies $\bigwedge_{\substack{c \in[x]_{\Theta} \\ d \in[y]_{\Theta}}} \chi_{A}(c \gamma d) \geq$ $\left(\bigwedge_{c \in[x]_{\Theta}} \chi_{A}(c)\right) \vee\left(\bigwedge_{d \in[y]_{\Theta}} \chi_{A}(d)\right)$. 
Case 2: $[x]_{\Theta} \not \subset A$ and $[y]_{\Theta} \not \subset A$. Then $\bigwedge_{c \in[x]_{\Theta}} \chi_{A}(c)=\bigwedge_{d \in[y]_{\Theta}} \chi_{A}(d)=0$. Hence $\bigwedge_{\substack{c \in[x]_{\Theta} \\ d \in[y]_{\Theta}}} \chi_{A}(c \gamma d) \geq\left(\bigwedge_{c \in[x]_{\Theta}} \chi_{A}(c)\right) \vee\left(\bigwedge_{d \in[y]_{\Theta}} \chi_{A}(d)\right)$.

We have

$$
\begin{aligned}
\underline{\Theta}\left(\chi_{A}\right)(x \gamma y) & =\bigwedge_{a \in[x \gamma y]_{\Theta}} \chi_{A}(a) \\
& =\bigwedge_{a \in[x]_{\Theta} \gamma[y]_{\Theta}} \chi_{A}(a) \\
& =\bigwedge_{\substack{c \in[x]_{\Theta} \\
d \in[y]_{\Theta}}} \chi_{A}(c \gamma d) \\
& \geq\left(\bigwedge_{c \in[x]_{\Theta}} \chi_{A}(c)\right) \vee\left(\bigwedge_{d \in[y]_{\Theta}} \chi_{A}(d)\right) \\
& =\underline{\Theta}\left(\chi_{A}\right)(x) \vee \underline{\Theta}\left(\chi_{A}\right)(y) .
\end{aligned}
$$

Therefore $\underline{\Theta}\left(\chi_{A}\right)$ is a fuzzy ideal of $S$.

Conversely, assume $\underline{\Theta}\left(\chi_{A}\right) \neq 0$ and $\underline{\Theta}\left(\chi_{A}\right)$ is a fuzzy ideal of $S$. Then there exists $a \in S$ such that $[a]_{\Theta} \subseteq A$. This implies $\underline{\Theta}(A) \neq \emptyset$. Let $x \in \underline{\Theta}(A)$ and $y \in S$. Then $[x]_{\Theta} \subseteq A$. So $\underline{\Theta}\left(\chi_{A}\right)(x)=\bigwedge_{c \in[x]_{\Theta}} \chi_{A}(c)=1$. Since $\underline{\Theta}\left(\chi_{A}\right)$ is a fuzzy ideal of $S$, we have $\underline{\Theta}\left(\chi_{A}\right)(x \gamma y) \geq \underline{\Theta}\left(\chi_{A}\right)(x)=1$. So $[x \gamma y]_{\Theta} \subseteq A$. Hence $x \gamma y \in \underline{\Theta}(A)$.

(ii) The proof of (ii) is similar to (i).

Theorem 4.4. Let $\Theta$ be a complete congruence on a $\Gamma$-semigroup $S$ and $f$ a fuzzy ideal of $S$. Then $\bar{\Theta}(f)$ is a fuzzy ideal of $S$.

Proof. Assume $f$ is a fuzzy ideal of $S$. Let $x, y \in S$ and $\gamma \in \Gamma$. Then $f(x \gamma y) \geq$ $f(x) \vee f(y)$. We have

$$
\begin{aligned}
\bar{\Theta}(f)(x \gamma y) & =\bigvee_{a \in[x \gamma y]_{\Theta}} f(a)=\bigvee_{a \in[x]_{\Theta} \gamma[y]_{\Theta}} f(a) \\
& =\bigvee_{\substack{c \in[x]_{\Theta} \\
d \in[y]_{\Theta}}} f(c \gamma d) \geq\left(\bigvee_{c \in[x]_{\Theta}} f(c)\right) \vee\left(\bigvee_{d \in[y]_{\Theta}} f(d)\right) \\
& =\bar{\Theta}(f)(x) \vee \bar{\Theta}(f)(y) .
\end{aligned}
$$

Then $\bar{\Theta}(f)(x \gamma y) \geq \bar{\Theta}(f)(x) \vee \bar{\Theta}(f)(y)$. Therefore $\bar{\Theta}(f)$ is a fuzzy ideal of $S$.

From Example 4.1, we can see that the converse of Theorem 4.4 is not true in general.

A fuzzy ideal $f$ of a $\Gamma$-semigroup $S$ is called a fuzzy prime ideal of $S$ if $f(x \gamma y)=f(x)$ or $f(x \gamma y)=f(y)$ for all $x, y \in S$ and $\gamma \in \Gamma$. 
Theorem 4.5. Let $f$ be a fuzzy subset of a $\Gamma$-semigroup $S$. Then $f$ is a fuzzy prime ideal of $S$ if and only if for all $\lambda \in[0,1]$, if $f_{\lambda} \neq \emptyset$, then $f_{\lambda}$ is a prime ideal of $S$.

Proof. Assume $f$ is a fuzzy prime ideal of $S$. Then $f$ is a fuzzy ideal of $S$. Assume $f_{\lambda} \neq \emptyset$. By Theorem 4.1, $f_{\lambda}$ is an ideal of $S$. Let $x, y \in S$ and $\gamma \in \Gamma$ such that $x \gamma y \in f_{\lambda}$. Since $f$ is a fuzzy prime ideal of $S, f(x \gamma y)=f(x)$ or $f(x \gamma y)=f(y)$. This implies $x \in f_{\lambda}$ or $y \in f_{\lambda}$. Therefore $f_{\lambda}$ is a prime ideal of $S$.

Conversely, assume for all $\lambda \in[0,1]$, if $f_{\lambda} \neq \emptyset$, then $f_{\lambda}$ is a prime ideal of $S$. Let $x, y \in S$ and $\gamma \in \Gamma$. By Theorem 4.1, $f$ is a fuzzy ideal of $S$. This implies $f(x \gamma y) \geq f(x)$ and $f(x \gamma y) \geq f(y)$. Let $\lambda=f(x \gamma y)$. Thus $x \gamma y \in f_{\lambda}$. Since $f_{\lambda}$ is a prime ideal of $S, x \in f_{\lambda}$ or $y \in f_{\lambda}$. This implies that $f(x) \geq \lambda=f(x \gamma y)$ or $f(y) \geq \lambda=f(x \gamma y)$. Hence $f(x \gamma y)=f(x)$ or $f(x \gamma y)=f(y)$. Hence $f$ is a fuzzy prime ideal of $S$.

Theorem 4.6. Let $f$ be a fuzzy subset of a $\Gamma$-semigroup $S$. Then $f$ is a fuzzy prime ideal of $S$ if and only if for all $\lambda \in[0,1]$, if $f_{\lambda}^{S} \neq \emptyset$, then $f_{\lambda}^{S}$ is a prime ideal of $S$.

Proof. Assume $f$ is a fuzzy prime ideal of $S$. Then $f$ is a fuzzy ideal of $S$. Assume $f_{\lambda}^{S} \neq \emptyset$. By Theorem 4.2, $f_{\lambda}^{S}$ is an ideal of $S$. Let $x, y \in S$ and $\gamma \in \Gamma$ such that $x \gamma y \in f_{\lambda}^{S}$. Then $f(x \gamma y)>\lambda$. Since $f$ is a fuzzy prime ideal of $S$, $f(x \gamma y)=f(x)$ or $f(x \gamma y)=f(y)$. This implies $f(x)>\lambda$ or $f(y)>\lambda$. Hence $x \in f_{\lambda}^{S}$ or $y \in f_{\lambda}^{S}$. Therefore $f_{\lambda}^{S}$ is a prime ideal of $S$.

Conversely, assume for all $\lambda \in[0,1]$, if $f_{\lambda}^{S} \neq \emptyset$, then $f_{\lambda}^{S}$ is a prime ideal of $S$. Let $x, y \in S$ and $\gamma \in \Gamma$. By Theorem 4.2, $f$ is a fuzzy ideal of $S$. This implies $f(x \gamma y) \geq f(x)$ and $f(x \gamma y) \geq f(y)$. We have $x \gamma y \in f_{\lambda}^{S}$ for all $\lambda<f(x \gamma y)$. Since $f_{\lambda}^{S}$ is a prime ideal of $S$ for all $\lambda<f(x \gamma y), x \in f_{\lambda}^{S}$ or $y \in f_{\lambda}^{S}$ for all $\lambda<f(x \gamma y)$. This implies that $f(x)>\lambda$ or $f(y)>\lambda$ for all $\lambda<f(x \gamma y)$. Then $f(x) \geq f(x \gamma y)$ or $f(y) \geq f(x \gamma y)$. Hence $f(x \gamma y)=f(x)$ or $f(x \gamma y)=f(y)$. Hence $f$ is a fuzzy prime ideal of $S$.

Let $\Theta$ be a congruence on a $\Gamma$-semigroup $S$. A fuzzy subset $f$ of $S$ is called a $\Theta$-lower rough fuzzy prime ideal of $S$ if $\underline{\Theta}(f)$ is a fuzzy prime ideal of $S$. A $\Theta$-upper rough fuzzy prime ideal of $S$ is defined analogously. We call $f$ a rough fuzzy prime ideal of $S$ if it is both a $\Theta$-lower and $\Theta$-upper rough fuzzy prime ideal of $S$.

Lemma 4.7. Let $\Theta$ be a congruence on a $\Gamma$-semigroup $S$. If $f$ is a fuzzy subset of $S$ and $\lambda \in[0,1]$, then

(i) $](\underline{\Theta}(f))_{\lambda}=\underline{\Theta}\left(f_{\lambda}\right)$ and

(ii) $(\bar{\Theta}(f))_{\lambda}^{S}=\bar{\Theta}\left(f_{\lambda}^{S}\right)$.

Proof. (i) Let $x \in(\underline{\Theta}(f))_{\lambda}$. Then $\underline{\Theta}(f)(x) \geq \lambda$. So $\bigwedge_{a \in[x]_{\Theta}} f(a) \geq \lambda$. Therefore $f(a) \geq \lambda$ for all $a \in[x]_{\Theta}$. This implies $[x]_{\Theta} \subseteq f_{\lambda}$. Therefore $x \in \underline{\Theta}\left(f_{\lambda}\right)$. 
Conversely, assume $x \in \underline{\Theta}\left(f_{\lambda}\right)$. Thus $[x]_{\Theta} \subseteq f_{\lambda}$. Then $f(a) \geq \lambda$ for all $a \in[x]_{\Theta}$. This implies $\bigwedge_{a \in[x]_{\Theta}} f(a) \geq \lambda$. Thus $\underline{\Theta}(f)(x) \geq \lambda$. Hence $x \in(\underline{\Theta}(f))_{\lambda}$.

(ii) Let $x \in(\bar{\Theta}(f))_{\lambda}^{S}$. Then $\bar{\Theta}(f)(x)>\lambda$. So $\bigvee_{a \in[x]_{\Theta}} f(a)>\lambda$. Therefore $f(a)>\lambda$ for some $a \in[x]_{\Theta}$. This implies $[x]_{\Theta} \cap f_{\lambda}^{S} \neq \emptyset$. Therefore $x \in \bar{\Theta}\left(f_{\lambda}^{S}\right)$.

Conversely, assume $x \in \bar{\Theta}\left(f_{\lambda}^{S}\right)$. Thus $[x]_{\Theta} \cap f_{\lambda}^{S} \neq \emptyset$. Then $f(a)>\lambda$ for some $a \in[x]_{\Theta}$. This implies $\bigvee_{a \in[x]_{\Theta}} f(a)>\lambda$. Thus $\bar{\Theta}(f)(x)>\lambda$. Hence $x \in(\bar{\Theta}(f))_{\lambda}^{S}$.

Theorem 4.8. Let $f$ be a fuzzy prime ideal of a $\Gamma$-semigroup $S$ and $\Theta$ be a complete congruence on $S$. Then $f$ is a rough fuzzy prime ideal of $S$.

Proof. Let $f$ be a fuzzy prime ideal of a $\Gamma$-semigroup $S$ and $\Theta$ a complete congruence on $S$. By Theorem 4.5, for all $\lambda \in[0,1]$, if $f_{\lambda} \neq \emptyset$, then $f_{\lambda}$ is a prime ideal of $S$. By Theorem 3.1(i), for all $\lambda \in[0,1]$, if $\underline{\Theta}\left(f_{\lambda}\right) \neq \emptyset$, then $\underline{\Theta}\left(f_{\lambda}\right)$ is a prime ideal of $S$. From this and Lemma 4.7(i), for all $\lambda \in[0,1]$, if $(\underline{\Theta}(f))_{\lambda} \neq \emptyset,(\underline{\Theta}(f))_{\lambda}$ is a prime ideal of $S$. By Theorem 4.5, $\underline{\Theta}(f)$ is a fuzzy prime ideal of $S$. Hence $f$ is a $\Theta$-lower rough fuzzy prime ideal of $S$. Similarly, $f$ is a $\Theta$-upper rough fuzzy prime ideal of $S$. Therefore $f$ is a rough fuzzy prime ideal of $S$.

Theorem 4.9. Let $\Theta$ be a congruence on a $\Gamma$-semigroup $S$. Then $f$ is a $\Theta$ lower rough fuzzy prime ideal if and only if for all $\lambda \in[0,1]$, if $\underline{\Theta}\left(f_{\lambda}\right) \neq \emptyset$, then $f_{\lambda}$ is a $\Theta$-lower rough prime ideal of $S$.

Proof. By Theorem 4.5 and Lemma 4.7(i), we can obtain the conclusion easily.

Theorem 4.10. Let $\Theta$ be a congruence on a $\Gamma$-semigroup $S$. Then $f$ is a $\Theta$-upper rough fuzzy prime ideal if and only if for all $\lambda \in[0,1]$, if $f_{\lambda}^{S} \neq \emptyset$, then $f_{\lambda}^{S}$ is a $\Theta$-upper rough prime ideal of $S$.

Proof. By Theorem 4.6 and Lemma 4.7(ii), we can obtain the conclusion easily.

Acknowledgements. The author would like to thank the referees for helpful suggestion and the Commission on Higher Education and the Thailand Research Fund (TRF) for grant support.

\section{References}

[1] M. Banerjee and S. K. Pal, Roughness of a fuzzy set, Information Science 93 (1996), no. $3-4,235-246$.

[2] R. Biswas and S. Nanda, Rough groups and rough subgroups, Bulletin of the Polish Academy of Sciences, Mathematics 42 (1994), no. 3, 251-254. 
[3] R. Chinram, On quasi-gamma-ideals in gamma-semigroups, ScienceAsia 32 (2006), no. 4, 351-353.

[4] R. Chinram and C. Jirojkul, On bi- $\Gamma$-ideals in $\Gamma$-semigroups, Songklanakarin Journal of Science and Technology 29 (2007), no. 1, 231-234.

[5] R. Chinram and P. Siammai, On green's relations for $\Gamma$-semigroups and reductive $\Gamma$ semigroups, International Journal of Algebra 2 (2008), no. 4, 187-195.

[6] B. Davvaz, Rough subpolygroups in a factor polygroup, Journal of Intelligent and Fuzzy Systems 17 (2006), no. 6, 613-621.

[7] J. Iwinski, Algebraic approach to rough sets, Bulletin of the Polish Academy of Sciences, Mathematics 35 (1987), 673-683.

[8] Y. B. Jun, Roughness of gamma-subsemigroups/ideals in gamma-semigroups, Bull. Korean Math. Soc. 40 (2003), no. 3, 531-536.

[9] - On closure gamma-semigroups, Commun. Korean Math. Soc. 19 (2004), no. 4, 639-641.

[10] N. Kuroki, Fuzzy congruences and fuzzy normal subgroups, Information Sciences 60 (1992), no. 3, 247-259.

[11] _ Rough ideals in semigroups, Information Science 100 (1997), no. 1-4, 139-163.

[12] N. Kuroki and P. P. Wang, The lower and upper approximations in a fuzzy group, Information Science 90 (1996), no. 1-4, 203-220.

[13] Y. I. Kwon and S. K. Lee, On weakly prime ideals of ordered $\Gamma$-semigroups, Commun. Korean Math. Soc. 13 (1998), no. 2, 251-256.

[14] Z. Pawlak, Rough sets, International Journal of Computer and Information Sciences 11 (1982), 341-356.

[15] N. K. Saha, On Г-semigroup II, Bull. Calcutta Math. Soc. 79 (1987), no. 6, 331-335.

[16] On Г-semigroup III, Bull. Calcutta Math. Soc. 80 (1988), no. 1, 1-12.

[17] M. K. Sen, On Г-semigroup, Algebra and its applications (New Delhi, 1981), 301-308, Lecture Note in Pure and Appl. Math., 91, Dekker, New York, 1984.

[18] M. K. Sen and N. K. Saha, On Г-semigroup I, Bull. Calcutta Math. Soc. 78 (1986), 181-186.

[19] M. Siripitukdet and A. Iampan, On the ordered $n$-prime ideals in ordered $\Gamma$-semigroups, Commun. Korean Math. Soc. 23 (2008), no. 1, 19-27.

[20] Q. M. Xiao and Z. L. Zhang, Rough prime ideals and rough fuzzy prime ideals in semigroups, Information Sciences 176 (2006), no. 6, 725-733.

Department of Mathematics

FACUlty of Science

Prince of Songkla University

Hat Yai, Songkhla 90112, Thailand

E-mail address: ronnason.c@psu.ac.th 